\title{
Comparative Analysis of Spontaneous Infectious Spondylitis : Pyogenic versus Tuberculous
}

\author{
Yangwon Lee, M.D., Bum-Joon Kim, M.D., Ph.D., Se-Hoon Kim, M.D., Ph.D., Seung-Hwan Lee, M.D., Won-Hyung Kim, M.D., \\ Sung-Won Jin, M.D. \\ Department of Neurosurgery, Korea University Ansan Hospital, Ansan, Korea
}

Objective : Spondylitis is often chemotherapy resistant and requires long-term treatment. Without adequate chemotherapy, the outcome can be fatal or result in severe neurologic damage. Therefore, differentiating the etiology of spondylitis is very important, particularly in spontaneous cases. As the prevalence of tuberculosis in Korea has decreased in recent years, updated clinical research about spondylitis is warranted.

Methods : From April 2010 to March 2016, data from spondylitis patients were collected retrospectively. In total, 69 patients (51 with pyogenic spondylitis and 18 with tuberculous spondylitis) were included. Clinical data, laboratory findings including erythrocyte sedimentation rate (ESR) and C-reactive protein (CRP) level, measurements of Cobb angles at the initial and final followup, and radiologic features on magnetic resonance imaging (MRI) scans were evaluated. To test differences between the pyogenic and tuberculous groups, numerical data were compared using the student's t-test and Mann-Whitney U test, and categorical data were compared using the chi-square test and Fisher's exact test.

Results : The patients' mean age was 60.0 years. Male sex was slightly predominant (56.5\%). There was no difference in mean age and sex between the two groups. The pyogenic group had a relatively higher proportion of immunocompromised patients. The peak CRP value was higher in the pyogenic group than in the tuberculous group $(14.08 \mathrm{mg} / \mathrm{dL}$ and $8.50 \mathrm{mg} / \mathrm{dL}$, respectively, $p=0.009)$, whereas the ESR was not significantly different between the groups $(81.5 \mathrm{~mm} / \mathrm{h}$ and $75.6 \mathrm{~mm} / \mathrm{h}$, respectively, $p=0.442)$. Radiologically, the presence of disc space sparing and vertebral body collapse differed between the groups. In the tuberculous group, the disc was more commonly preserved on contrast-enhanced MRI ( $50 \%$ and $23.5 \%$, respectively, $p=0.044)$, and vertebral body collapse was more common (66.6\% and $15.7 \%$, respectively, $p<0.001)$. The mean length of hospitalization was longer in the pyogenic group (56.5 days and 41.2 days, respectively, $p=0.001$ ). Four mortality cases were observed only in the pyogenic group. The most commonly isolated microorganism in the pyogenic group was Staphylococcus aureus (S. aureus) (methicillin susceptible S. aureus and methicillin resistant $S$. aureus [MRSA] in 8 and 4 cases, respectively).

Conclusion : The clinical and radiological manifestations of spontaneous spondylitis differ based on the causative organism. Pyogenic spondylitis patients tend to have a higher CRP level and a more severe clinical course, whereas tuberculous spondylitis patients present with destruction of the vertebral body with disc sparing more frequently. The presence of MRSA is increasing in community-acquired spondylitis cases.

Key Words : Spondylitis · Osteomyelitis · Bone diseases, Infectious · Bacterial infection · Spondylodiscitis · Tuberculosis.

- Received : December 23, 2016 •Revised : April 13, 2017 •Accepted : April 19, 2017

- Address for reprints : Bum-Joon Kim, M.D., Ph.D.

Department of Neurosurgery, Korea University Ansan Hospital, 123 Jeokgeum-ro, Danwon-gu, Ansan 15355, Korea

Tel : +82-31-412-5053, Fax : +82-31-412-5054, E-mail : nsbjkim@gmail.com

This is an Open Access article distributed under the terms of the Creative Commons Attribution Non-Commercial License (http://creativecommons.org/licenses/by-nc/4.0) which permits unrestricted non-commercial use, distribution, and reproduction in any medium, provided the original work is properly cited. 


\section{INTRODUCTION}

Spondylitis refers to osteomyelitis that arises in the spine. In many cases, spondylitis patients respond poorly to antimicrobial chemotherapy, and even if they do respond to treatment, complete remission takes a long time ${ }^{20)}$. Therefore, early initiation of chemotherapy is very important to achieve better results and to reduce the neurological sequelae and socioeconomic burden. In many cases, the causative microorganism cannot be isolated. Having a general idea of the most likely causative microorganism is important for empirical chemotherapy of spondylitis. If the patient does not show any definite source of infection such as a preceding infection, history of surgery, prosthesis insertion, or trauma, the spondylitis is defined as spontaneous spondylitis.

Historically, in spontaneous spondylitis, the first step is to differentiate between pyogenic and tuberculous spondylitis. Many authors have studied the differences between pyogenic and tuberculous spondylitis ${ }^{3,5,16,24)}$. South Korea had many tuberculosis patients and public health worsened after the Korean War. However, recently, the prevalence of tuberculosis in Korea has markedly decreased because of vaccination and adequate chemotherapy ${ }^{12,18)}$. The microorganisms that affect the spine are continuously changing ${ }^{23)}$. Several antibiotic-resistant microorganisms have arisen, and new antibiotics have been developed to address this problem.

We hypothesize that the manifestations of recent spontaneous spondylitis cases would differ from those reported in the past and believe that updated clinical research on this subject is warranted. Here, we present our retrospective study of patients with spontaneous spondylitis during the last 6 years.

\section{MATERIALS AND METHODS}

\section{Patients and methods}

From April 2010 to March 2016, the medical records and radiographs of patients with a diagnosis of spondylitis or spondylodiscitis, who admitted to our department, were retrospectively reviewed. The inclusion criteria were diagnosis of spondylitis by magnetic resonance imaging (MRI), availability of radiologic and laboratory study data, and a minimum clinical follow-up of 6 months. There were 135 patients who met these criteria, among whom 66 patients were excluded for the following reasons: spine surgery or prosthetic device insertion within the last 5 years, invasive procedures such as nerve block and acupuncture within the last year, and follow-up loss before the end of antibiotic treatment. The remaining 69 patients were divided into two groups according to the causative microorganism (51 patients with pyogenic spondylitis and $18 \mathrm{pa}-$ tients with tuberculous spondylitis) and were analyzed.

\section{Acquisition of laboratory and radiologic data}

In all patients, the erythrocyte sedimentation rate (ESR), Creactive protein (CRP) level, and white blood cell (WBC) count at the start of and during antibiotics treatment were evaluated at least once a week. For statistical comparison, the peak ESR, CRP, and WBC values were collected. The proportion of patients with high peak values of inflammatory markers $(E S R>100 \mathrm{~mm} / \mathrm{h}$ and CRP $>10 \mathrm{mg} / \mathrm{dL})$ and the proportion of neutrophils were compared between the two groups. To assess the quantity of vertebral body destruction and development of deformity, plain radiographs were taken at the start of antibiotics treatment and every week thereafter. Based on previous studies, bone destruction may not be observed in the first 13 weeks ${ }^{14,20)}$; therefore, the Cobb angle of the involved vertebral bodies was measured using plain radiographs at the initial diagnosis and at least 2 months after the end of antibiotics treatment. The difference between the initial and final Cobb angle was calculated and compared between the two groups. MRI was performed for the diagnosis of spondylitis and at 4 to 6 weeks after antibiotics therapy. MRI findings were also reviewed and collected to determine the differences in radiologic features between both groups.

\section{Statistical analysis}

Statistical analyses were performed using SPSS Statistics version 20.0 software (IBM Corp., Armonk, NY, USA). Comparison of numerical data were performed using the student's t-test except for peak ESR, peak CRP, and the number of involved vertebral bodies that did not have a normal distribution on Kolmogorov-Smirnov and Shapiro-Wilk tests. Peak ESR, peak CRP values, peak WBC counts, the proportion of neutrophils, and the number of involved vertebral bodies were compared using the Mann-Whitney U test. For categorical data, underlying diseases were compared using the Fisher's exact test, and the others were compared using the chi-square 
test. A $p$ value $<0.05$ was considered statistically significant.

\section{RESULTS}

A total of 69 patients ( 39 males and 30 females) with a mean age of 60.0 (8-83) years were included. The characteristics of pyogenic and tuberculous spondylitis at the time of diagnosis are listed in Table 1. The mean age of patients was 60.6 (22-83) and $57.0(8-83)$ years in the pyogenic and tuberculous groups, respectively. The pyogenic group showed male predominance (32 males and 19 females), whereas the tuberculous group showed female predominance (7 males and 11 famales). Regarding the medical history, in the pyogenic group, the most frequent underlying diseases and conditions were diabetes, chronic renal failure, liver cirrhosis, steroid use, and alcohol abuse. Particularly, five patients with chronic renal failure were identified in the pyogenic group, while none was identified in the tuberculous group.

Regarding laboratory data, the mean values of inflammatory markers were higher in the pyogenic group than in the tuberculous group. The peak ESR was $81.5 \mathrm{~mm} / \mathrm{h}$ and $75.6 \mathrm{~mm} / \mathrm{h}$ in the pyogenic and tuberculous groups, respectively. The peak CRP level was significantly higher in the pyogenic group than in the tuberculous group $(14.08 \mathrm{mg} / \mathrm{dL}$ and $8.50 \mathrm{mg} / \mathrm{dL}$, respectively, $p=0.009$ ). The percentage of patients with high ESR $(>100 \mathrm{~mm} / \mathrm{h})$ and high CRP level $(>10 \mathrm{mg} / \mathrm{dL})$ was slightly higher in the pyogenic group $(p>0.05)$. The peak WBC counts were significantly higher in the pyogenic group than in the tuberculous group $\left(12.77 \times 10^{3} / \mu \mathrm{L}\right.$ and $9.23 \times 10^{3} / \mu \mathrm{L}$, respectively, $p=0.002)$. However, the proportion of neutrophils was not different between the two groups $(81.1 \%$ and $75.1 \%$, respectively, $p=0.132$ ).

The mean length of hospitalization was 15.3 days longer in the pyogenic group ( $p=0.001$ ). Twenty-nine and 10 patients in the pyogenic and tuberculous groups, respectively, underwent surgical treatment. Abscess removal was more common in the pyogenic group, whereas stabilization was more common in the tuberculous group $(p>0.05)$. Combined infectious diseases such as pneumonia, urinary tract infection, and sepsis were identified only in the pyogenic group. Four mortality cases were found only in the pyogenic group $(p<0.001)$.

The comparison of radiographic parameters is shown in Table 2. The average number of affected vertebral bodies was
2.53 and 2.18 in the pyogenic and tuberculous groups, respectively ( $p=0.256$ ). The most frequently affected vertebra was the lumbar vertebra in both groups, followed by the thoracolumbar (T11-L1) vertebra in the pyogenic group and the thoracic vertebra in the tuberculous group; however, there were no significant between-group differences (Table 2). The proportion of patients with vertebral body collapse was signifi-

Table 1. Characteristics of patients with spontaneous spondylitis

\begin{tabular}{|c|c|c|c|}
\hline & Pyogenic & Tuberculous & $p$-value \\
\hline Total & 51 & 18 & \\
\hline Male & $32(62.7)$ & $7(38.9)$ & 0.101 \\
\hline Mean age (years) & $60.6(22-83)$ & $57.0(8-83)$ & 0.440 \\
\hline$\geq 60$ & $28(54.9)$ & $9(50.0)$ & \\
\hline$<60$ & $23(45.1)$ & $9(50.0)$ & \\
\hline Isolation of microorganism & $27(52.9)$ & $14(77.8)$ & 0.065 \\
\hline Medical history & $13(25.5)$ & $4(22.2)$ & 0.782 \\
\hline Diabetes & $9(17.6)$ & $3(16.7)$ & 1.000 \\
\hline Chronic renal failure & $5(9.8)$ & $0(0.0)$ & 0.316 \\
\hline Cancer & $1(2.0)$ & $1(5.6)$ & 1.000 \\
\hline Liver cirrhosis & $1(2.0)$ & $0(0.0)$ & 1.000 \\
\hline Steroid use & $1(2.0)$ & $0(0.0)$ & 1.000 \\
\hline Alcohol use & $1(2.0)$ & $0(0.0)$ & 1.000 \\
\hline \multicolumn{4}{|l|}{ Inflammatory marker } \\
\hline Peak ESR (mm/h) & $\begin{array}{c}81.5 \\
(22-121)\end{array}$ & $\begin{array}{c}75.6 \\
(26-119)\end{array}$ & 0.442 \\
\hline $\operatorname{ESR}(\geq 100 \mathrm{~mm} / \mathrm{h})$ & $16(31.4)$ & $5(27.8)$ & 0.880 \\
\hline Peak CRP (mg/dL) & $\begin{array}{c}14.08 \\
(0.06-38.00)\end{array}$ & $\begin{array}{c}8.50 \\
(0.75-24.00)\end{array}$ & $0.009^{*}$ \\
\hline $\mathrm{CRP}(\geq 10 \mathrm{mg} / \mathrm{dL})$ & $27(52.9)$ & $6(33.3)$ & 0.152 \\
\hline Peak WBCs $\left(\times 10^{3} / \mu \mathrm{L}\right)$ & 12.77 & 9.23 & $0.002^{*}$ \\
\hline Neutrophils (\%) & 81.1 & 75.1 & 0.132 \\
\hline $\begin{array}{l}\text { Length of hospitalization } \\
\text { (days) }\end{array}$ & $\begin{array}{c}56.5 \\
(13-202)\end{array}$ & $\begin{array}{c}41.2 \\
(8-192)\end{array}$ & $0.001^{*}$ \\
\hline Combined infection & $11(21.6)$ & $0(0.0)$ & $<0.001^{*}$ \\
\hline Sepsis & $6(11.8)$ & $0(0.0)$ & \\
\hline Pneumonia & $3(5.9)$ & $0(0.0)$ & \\
\hline Urinary tract infection & $2(3.9)$ & $0(0.0)$ & \\
\hline Surgery & $29(56.9)$ & $10(55.6)$ & 0.923 \\
\hline Abscess removal & $26(51.0)$ & $5(27.8)$ & 0.089 \\
\hline Stabilization & $16(31.4)$ & $8(44.4)$ & 0.317 \\
\hline Mortality & $4(7.8)$ & $0(0.0)$ & $<0.001^{*}$ \\
\hline
\end{tabular}

Values are presented as mean (range) or number (\%). * Indicates significant difference with $p<0.05$. ESR : erythrocyte sedimentation rate, CRP : C-reactive protein, WBC : white blood cell 
cantly higher in the tuberculous group than in the pyogenic group (66.7\% and $15.7 \%$, respectively, $p<0.001)$. The proportion of patients with disc sparing on MRI scans was significantly higher in the tuberculous group than in the pyogenic group (50\% and $23.5 \%$, respectively, $p=0.044$ ). Epidural abscess and psoas muscle abscess were more common in the pyogenic group, but the difference did not reach statistical significance. Regarding the measurement of the Cobb angle in the affected vertebral bodies on simple radiographs, the difference between the initial and final Cobb angle was $0.11^{\circ}$ in the pyogenic group and $1.46^{\circ}$ in the tuberculous group, respectively, and was not significantly different.

The causative organism was isolated in 27 (52.9\%) and 14 (77.8\%) patients in the pyogenic and tuberculous groups, respectively (Table 1). The isolated organisms are listed in Table 3. The most common microorganism in the pyogenic group was Staphylococcus aureus (S. aureus). S. aureus was identified in 12 patients (methicillin-sensitive S. aureus [MSSA] in 8 pa-

Table 2. Radiologic parameters of spontaneous spondylitis

\begin{tabular}{|c|c|c|c|}
\hline & Pyogenic & Tuberculous & $p$-value \\
\hline Number of affected VB & $2.53(1-9)$ & $2.18(1-3)$ & 0.256 \\
\hline 1 & $1(2.0)$ & $1(5.6)$ & \\
\hline 2 & $33(64.7)$ & $13(72.2)$ & \\
\hline 3 & $13(25.5)$ & $4(22.2)$ & \\
\hline Over 4 & $4(7.8)$ & $0(0.0)$ & \\
\hline Location of affected VB & & & 0.160 \\
\hline Cervical & $2(3.9)$ & $1(5.6)$ & \\
\hline Thoracic (T1-T10) & $6(11.8)$ & $6(33.3)$ & \\
\hline Thoracolumbar (T11-L1) & $13(25.5)$ & $4(22.2)$ & \\
\hline Lumbar & $30(58.5)$ & $7(38.9)$ & \\
\hline \multicolumn{4}{|l|}{ MRI findings } \\
\hline VB collapse & $8(15.7)$ & $12(66.7)$ & $<0.001^{*}$ \\
\hline Disc sparing & $12(23.5)$ & $9(50.0)$ & $0.044^{*}$ \\
\hline Epidural abscess & $28(54.9)$ & $6(33.3)$ & 0.171 \\
\hline Psoas muscle abscess & $41(80.4)$ & $10(55.6)$ & 0.060 \\
\hline \multicolumn{4}{|l|}{$\begin{array}{l}\text { Cobb angle on radiograph } \\
\text { (degrees) }\end{array}$} \\
\hline Initial & $14.1(0.8-48.6)$ & $14.9(0.5-35.3)$ & 0.636 \\
\hline Final & $14.2(0.2-38.5)$ & $16.3(1.8-39.3)$ & 0.634 \\
\hline Alteration & 0.11 & 1.46 & 0.498 \\
\hline
\end{tabular}

Values are presented as mean (range) or number (\%). ${ }^{*}$ Indicates significant difference with $p<0.05$. VB : vertebral body, MRI : magnetic resonance imaging tients and methicillin-resistant $S$. aureus [MRSA] in 4 patients). Twenty-four patients suspected to have pyogenic spondylitis were treated with empirical antibiotics. Eleven (45.8\%) patients were treated with vancomycin or teicoplanin, 10 (41.7\%) patients were treated with first-generation cephalosporins, and the remaining 3 patients were treated with other cephalosporins and metronidazole. Among the 14 proven tuberculous spondylitis patients, the causative microorganism was isolated using bone biopsy in 11 patients and using sputum culture or aspiration of the psoas abscess in the remaining 3 patients. Four patients with negative culture were treated with tuberculosis medications after consultation with the division of infectious diseases. Three of them showed MRI findings that were highly suggestive of tuberculous spondylitis and yielded positive results on tuberculin test or had a history of pulmonary tuberculosis. The one remaining patient was empirically diagnosed with tuberculosis after the failure of antibiotics treatment for pyogenic spondylitis.

Table 3. Isolated organisms of spontaneous spondylitis

\begin{tabular}{lc}
\hline Microbiology & Value \\
\hline Pyogenic spondylitis & 51 \\
Staphylococcus aureus & $12(23.5)$ \\
MSSA & $8(15.7)$ \\
MRSA & $4(7.8)$ \\
Escherichia coli & $4(7.8)$ \\
Coagulase-negative Staphylococcus & $3(5.9)$ \\
Enterococcus & $2(3.9)$ \\
Streptococcus spp. & $2(3.9)$ \\
Pseudomonas aeruginosa & $1(2.0)$ \\
Acinetobacter & $1(2.0)$ \\
Enterobacteriaceae & $1(2.0)$ \\
Stenotrophomonas maltophilia & $1(2.0)$ \\
Negative & $24(47.1)$ \\
Tuberculous spondylitis & 18 \\
(Mycobacterium tuberculosis) & \\
Proven using bone biopsy & $11(61.1)$ \\
Proven using sputum culture & $2(11.1)$ \\
Proven using abscess aspiration & $1(5.6)$ \\
Negative & $4(22.2)$ \\
\hline
\end{tabular}

Values are presented as number (\%). MRSA : methicillin resistant Staphylococcus aureus, MSSA : methicillin sensitive Staphylococcus aureus 


\section{DISCUSSION}

Differences between pyogenic and tuberculous spondylitis in terms of clinical manifestations and radiologic findings were found in the present study. The prevalence of tuberculosis in South Korea has decreased in the last 30 years owing to improvements in nutrition and compliance, the National vaccination program, and the development of chemotherapies ${ }^{12,18)}$. However, the prevalence is still higher than that in other developed countries ${ }^{18)}$. Therefore, Korean spine clinicians always consider the possibility of tuberculous spondylitis in clinical practice. The present retrospective review suggests that the prevalence of tuberculous spondylitis is decreasing. Pyogenic spondylitis can arise from hematogenous spread of microorganisms from the skin, respiratory tract, genitourinary tract, gastrointestinal tract, and oral cavity ${ }^{4}$. Once the patient develops spondylitis, the elimination of the infection takes a very long time and can be fatal, so the proper choice of antibiotics is important. According to the 2015 Infectious Diseases Society of America (IDSA) guideline for spondylitis, unless the patient' $\mathrm{s}$ condition is deteriorating rapidly, it is better to obtain a microbiological diagnosis before starting empirical chemothera$\mathrm{py}^{2}$. Isolation of the virulent microorganism, using either bone biopsy or blood culture samples, is the most important step in the treatment of spondylitis ${ }^{19,20)}$. In spondylitis cases, computed tomography (CT)-guided bone biopsy is the diagnostic procedure of choice ${ }^{20)}$. In our department, CT-guided bone biopsy and blood culture were attempted for every patient with spondylitis. In patients with a paraspinal abscess pocket large enough to be aspirated, as shown by MRI, aspiration of the abscess for microbiologic diagnosis was also performed.

According to previous guidelines, chemotherapy should be maintained for 6 weeks for most bacterial spondylitis cases and for 3 months for Brucella-related spondylitis ${ }^{2}$. Though the criteria for the cessation of antibiotics for pyogenic spondylitis vary in each clinic, most studies suggest basing the decision on the improvement of pain and normalization of inflammatory markers ${ }^{7}$. In our series, intravenous antibiotics were administered for at least 6 weeks in most patients. If the inflammatory marker levels were elevated or not changed after 4 weeks of chemotherapy, the antibiotics were changed and therapy was maintained until the CRP level was normalized. Forty eight of the 51 patients with pyogenic spondylitis received chemotherapy for 6 to 9 weeks. One patient with microbiologically nega- tive results recovered after treatment for 3 months. There were two mortality cases due to septic shock; one of them was a 61-year-old woman in whom Streptococcus gallolyticus subspecies pasteurianus was isolated and who died at 4 weeks of treatment. The other patient was a 79-year-old man with Stenotrophomonas maltophilia who died after 6 months of treatment. $S$. aureus is known to be the most common microorganism associated with any type of osteomyelitis ${ }^{20)}$. MRSA has risen as an important pathogen also in community-acquired osteomyelitis $^{22)}$. In the present study, four patients with spontaneous spondylitis were found to be infected with MRSA (Table 3). This trend is essential when considering empirical chemotherapy. Five patients were transferred to our hospital because spondylitis could not be controlled in spite of chemotherapy for 1 or 2 weeks at other hospitals. All of them were treated with vancomycin or teicoplanin at our hospital for another 6 weeks. In our series, delayed recurrence of spondylitis did not occur after adequate chemotherapy was administered at our hospital.

The ESR and CRP level are deemed useful indicators by many clinicians in understanding infectious conditions including spondylitis. Zilkens et al. ${ }^{27)}$ suggested that among inflammation markers, the ESR shows a high sensitivity, while the CRP level demonstrates good diagnostic specificity for detecting spondylodiscitis. Previous studies have noted a higher CRP level and ESR in pyogenic spondylodiscitis than in tuberculous spondylodiscitis $^{13,17,26)}$. Lew and Waldvogel ${ }^{20)}$ suggested assessing the efficacy of antibiotics using CRP levels because the white blood cell count is not reliable, and the CRP level changes more rapidly than the ESR. In the present study, the mean CRP level was significantly higher in the pyogenic group, whereas the ESR did not show a significant difference between groups.

MRI is a useful tool for the diagnosis of spine infection. Spondylitis can be diagnosed in the early stage by endplate edema where T1 images show decreased signal intensity and T2 images show increased intensity ${ }^{1,15)}$ (Figs. 1 and 2). MRI is also a reliable method for assessing the epidural space and the spinal $\operatorname{cord}^{25)}$ with over $90 \%$ sensitivity, specificity, and accuracy ${ }^{1)}$. Owing to the absence of proteolytic enzymes of Mycobacterium tuberculosis (M. tuberculosis) and avascular characteristics of the disc, the disc space is usually preserved in tuberculous spondylitis until its nutritional support is affected ${ }^{8,15)}$. Previous studies have shown that tuberculous spine infection frequently 
spares disc involvement in MRI scans ${ }^{3,15)}$. However, in our series, the intervertebral disc was not affected in 12 patients in the pyogenic group, and in 11 of these 12 patients, multiple vertebral bodies were involved. While half of the patients in the tuberculous group showed disc sparing, the other half showed disc involvement. Therefore, we suggest that although the skipping pattern strongly suggests tuberculous infection, it cannot be a definite diagnosis.

The pattern on contrast-enhanced MRI is very important in the diagnosis of spondylitis ${ }^{3,22)}$. Tuberculous spondylitis tends to have a heterogeneous enhancement pattern of the vertebral body, whereas pyogenic spondylitis usually shows a homogenous pattern ${ }^{22)}$. Moreover, an abscess with rimmed enhancing walls can be visualized on MRI with contrast agents ${ }^{25)}$. In our series, 47 of the 51 patients showed contrast enhancement of the vertebral bodies at the end of antibiotic therapy even when the inflammatory markers were normalized. This finding suggests that the completion of chemotherapy does not require elimination of contrast enhancement on MRI.

On MRI, a psoas muscle abscess was observed in $80.4 \%$ $(41 / 51)$ and $55.5 \%(10 / 18)$ patients in the pyogenic and tuberculous groups, respectively (Table 2 ). If the paraspinal abscess is large, image-guided percutaneous abscess drainage is important to control infection and provide a microbiologic diagnosis. If the spondylitis is combined with an epidural abscess and the patient presents any neurologic symptoms, the patient should undergo surgical removal of the abscess and decompression of the neural structures. In the present study, 54.9\% (28/51) and $33.3 \%(6 / 18)$ patients in the pyogenic and tuberculous groups, respectively, had an epidural abscess and most underwent sur-
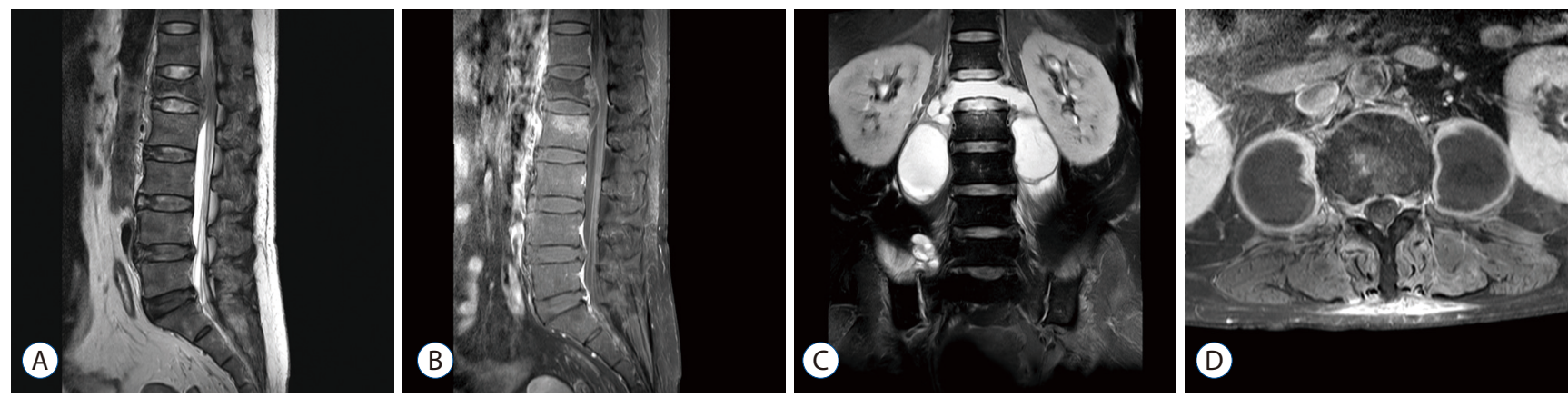

Fig. 1. Representative magnetic resonance imaging (MRI) scans of patients with methicillin resistant Staphylococcus aureus (MRSA) spondylitis. On the initial MRI scan of a 60-year-old woman who was diagnosed as having MRSA spondylitis, the L1 vertebral body was hypointense on a T2 weighted image and showed a bursting fracture with an epidural abscess compressing the conus medullaris (A). On a T1 image with contrast enhancement, the posterior wall of the L1 vertebra and upper endplate of the L2 vertebra showed strong enhancement, and the epidural abscess showed a rim-enhancing pattern (B). Despite abscess removal and vancomycin use, the follow-up MRI scan reveals that the L1 vertebral body had totally disappeared after 2 weeks, communicating with the paraspinal abscesses on both sides (C). Two $4.5 \times 4.0 \mathrm{~cm}$ sized abscess pockets were occupying the whole psoas muscles (D), which were removed by image-guided percutaneous drain insertion.
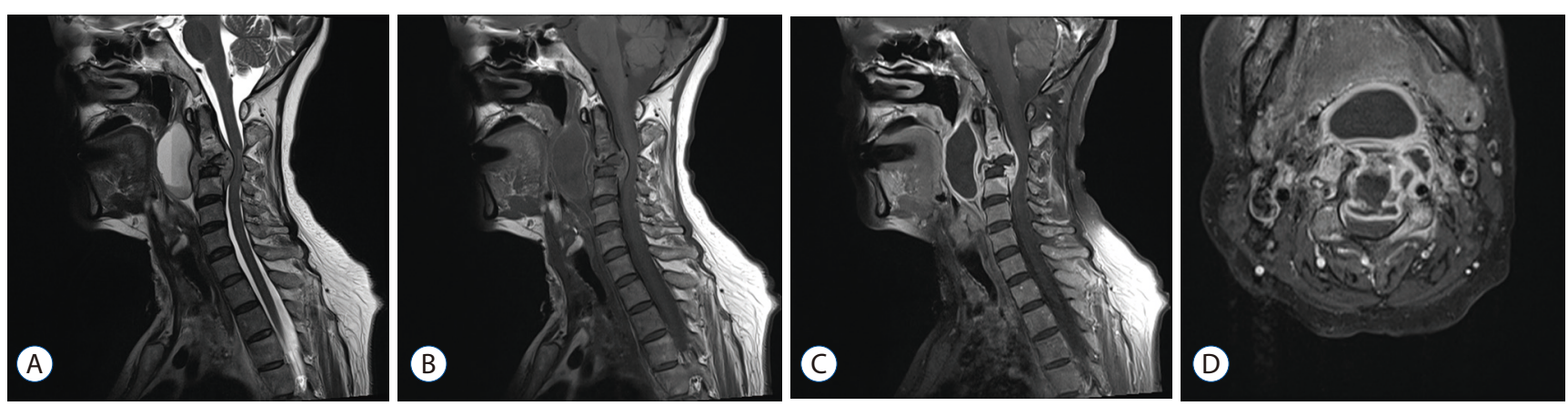

Fig. 2. Representative magnetic resonance images of tuberculous spondylitis. A 57-year old woman presented with hoarseness. A : A T2 sagittal image showed a destructive lesion at the C3 vertebral body compressing the spinal cord and a huge cystic lesion at the retropharyngeal space. Both the destroyed $\mathrm{C} 3$ vertebra and cyst were hypointense on $\mathrm{T} 1$ (B) and showed strong rim enhancement, whereas the disc spaces were relatively spared (C). On the axial image, the rim enhancing lesion of the vertebral body, epidural, and retropharyngeal space were communicating and severely encroaching the spinal canal (D). 
gery for evacuation of the epidural abscess. Sometimes, the decompressive surgery provides an important clue regarding the etiology of the spondylitis, such as caseating granuloma formation that suggests tuberculosis or purulent discharge, which in turn indicates bacterial infection.

Some spondylitis cases require spinal instrumentation because of the instability caused by the extensive bone destruction. The risk of biofilm formation exists, and the biofilm can make the eradication of the infection difficult ${ }^{6,9,10)}$. However, studies suggest that if the proper antibiotic is administered in the early stage of biofilm infection, it can be cured without removal of the medical device ${ }^{10}$. In 2016, in the IDSA guideline for tuberculosis, Nahid et al. ${ }^{21}$ suggested chemotherapy with rifampin for 6 to 9 months for tuberculous spondylitis and for 12 months in patients with instrumentation. In our series, $31.4 \%(16 / 51)$ and $44.4 \%(8 / 18)$ patients in the pyogenic and tuberculous groups, respectively, underwent instrumentation (Table 1). We suggest that if a patient shows spinal instability or if a patient is diagnosed with a bacterial infection, instrumentation should be postponed until after the infection is controlled to prevent biofilm formation. However, if the microorganism is not considered a biofilm-forming species such as $M$. tuberculosis ${ }^{11}$, the stabilization surgery does not need to be postponed.

In our review, vertebral body destruction in spondylitis was strongly associated with the type of organism. Two-thirds (12/18) of the patients showed destructive lesion in the tuberculous group (Fig. 2). Among the 8 cases with vertebral body destruction in the pyogenic group, the causative microorganism was S. aureus in 4 cases (2 MRSA and 2 MSSA) (Fig. 1). Though vertebral body destruction was more common in the tuberculous group, mortality was observed only in the pyogenic group. We suggest that tuberculous spondylitis and pyogenic spondylitis caused by $S$. aureus require more frequent radiologic follow-up to monitor the development of deformities.

In spontaneous spondylitis, both pyogenic and tuberculous spondylitis usually result from hematogenous spread ${ }^{4,820)}$. Thoracolumbar vertebrae have comparatively richer blood supply, and this area is more frequently involved in spondylitis cases. Because the segmental artery supplies two contiguous vertebral bodies, the hematogenous spread of spondylitis can affect multiple vertebral bodies even at the early stage of the disease ${ }^{4)}$. In 2010, Kim et al. ${ }^{17)}$ demonstrated that tuberculous spondylitis is associated with the involvement of over three levels. Howev- er, in our study, there was no difference in the number of affected levels. We suspect that with improvements in the early diagnosis of tuberculosis, the number of patients with extensive disease has decreased.

This study has some drawbacks. This is a retrospective study, and the data were collected from patients in a single neurosurgical department. The number of patients in the tuberculous spondylitis group was small. The number of tuberculosis cases in the general population has decreased; therefore, we rarely encounter tuberculosis cases in our department. Although patients with postoperative infection were not included in the present study (patients who underwent spine surgery or prosthetic device insertion within the last 5 years were excluded), the majority of spondylitis cases in our department were caused by postoperative infection. In contrast, the proportion of patients with severe predisposing medical conditions may have been underestimated; thus, the possibility of bias in this regard cannot be denied.

\section{CONCLUSION}

Spontaneous spondylitis can be diagnosed by clinical and radiologic findings. MRI patterns of disc sparing and vertebral body destruction suggest tuberculous spondylitis, whereas high CRP levels suggest pyogenic spondylitis. Because pyogenic spondylitis can be fatal, early isolation of the causative microorganism by bone biopsy or paraspinal aspiration, prevention of sepsis by adequate chemotherapy, and sequential follow-up of inflammatory markers are mandatory. For empirical chemotherapy, MRSA should be considered a pathogen.

\section{PATIENT CONSENT}

The patient provided written informed consent for the publication and the use of their images.

\section{References}

1. An HS, Seldomridge JA : Spinal infections: diagnostic tests and imaging studies. Clin Orthop Relat Res 444 : 27-33, 2006

2. Berbari EF, Kanj SS, Kowalski TJ, Darouiche RO, Widmer AF, Schmitt SK, 
et al. : Executive summary: 2015 Infectious Diseases Society of America (IDSA) clinical practice guidelines for the diagnosis and treatment of native vertebral osteomyelitis in adults. Clin Infect Dis $61: 859-863$, 2015

3. Chang MC, Wu HT, Lee CH, Liu CL, Chen TH : Tuberculous spondylitis and pyogenic spondylitis: comparative magnetic resonance imaging features. Spine (Phila Pa 1976) 31 : 782-788, 2006

4. Cheung W, Luk KD : Pyogenic spondylitis. Int Orthop 36 : 397-404, 2012

5. Colmenero JD, Jiménez-Mejías ME, Sánchez-Lora FJ, Reguera JM, Palomino-Nicás J, Martos F, et al. : Pyogenic, tuberculous, and brucellar vertebral osteomyelitis: a descriptive and comparative study of 219 cases. Ann Rheum Dis 56 : 709-715, 1997

6. Costerton JW, Stewart PS, Greenberg EP : Bacterial biofilms: a common cause of persistent infections. Science 284 : 1318-1322, 1999

7. Cottle L, Riordan T : Infectious spondylodiscitis. J Infect 56 : 401-412, 2008

8. De Backer Al, Mortelé KJ, Vanschoubroeck IJ, Deeren D, Vanhoenacker FM, De Keulenaer BL, et al. : Tuberculosis of the spine: $C T$ and MR imaging features. JBR-BTR 88 : 92-97, 2005

9. Donlan RM : Biofilms and device-associated infections. Emerg Infect Dis $7: 277-281,2001$

10. Høiby N, Bjarnsholt T, Moser C, Bassi GL, Coenye T, Donelli G, et al. : ESCMID guideline for the diagnosis and treatment of biofilm infections 2014. Clin Microbiol Infect 21 Suppl 1 : S1-S25, 2015

11. Ha KY, Chung YG, Ryoo SJ : Adherence and biofilm formation of staphylococcus epidermidis and mycobacterium tuberculosis on various spinal implants. Spine (Phila Pa 1976) 30 : 38-43, 2005

12. Hong YP, Kim SJ, Lew WJ, Lee EK, Han YC : The seventh nationwide tuberculosis prevalence survey in Korea, 1995. Int J Tuberc Lung Dis 2 : 27-36, 1998

13. Jeong SJ, Choi SW, Youm JY, Kim HW, Ha HG, Yi JS : Microbiology and epidemiology of infectious spinal disease. J Korean Neurosurg Soc $56: 21-27,2014$

14. Jevtic V : Vertebral infection. Eur Radiol 14 Suppl 3 : E43-E52, 2004

15. Joseffer SS, Cooper PR : Modern imaging of spinal tuberculosis. J Neu- rosurg Spine 2 : 145-150, 2005

16. Jung NY, Jee WH, Ha KY, Park CK, Byun JY : Discrimination of tuberculous spondylitis from pyogenic spondylitis on MRI. AJR Am J Roentgenol 182 : 1405-1410, 2004

17. Kim CJ, Song KH, Jeon JH, Park WB, Park SW, Kim HB, et al. : A comparative study of pyogenic and tuberculous spondylodiscitis. Spine (Phila Pa 1976) 35 : E1096-E1100, 2010

18. Kim HJ : Current status of tuberculosis in Korea. Korean J Med 82 : 257-262, 2011

19. Korean Society for Chemotherapy, Korean Society of Infectious Diseases, Korean Orthopaedic Association : Clinical guidelines for the antimicrobial treatment of bone and joint infections in Korea. Infect Chemother $46: 125-138,2014$

20. Lew DP, Waldvogel FA : Osteomyelitis. Lancet 364 : 369-379, 2004

21. Nahid P, Dorman SE, Alipanah N, Barry PM, Brozek JL, Cattamanchi A, et al. : Official American thoracic society/centers for disease control and prevention/infectious diseases society of America clinical practice guidelines: treatment of drug-susceptible tuberculosis. Clin Infect Dis 63 : e147-e195, 2016

22. Pigrau-Serrallach $C$, Rodríguez-Pardo D : Bone and joint tuberculosis. Eur Spine J 22 Suppl 4 : 556-566, 2013

23. Saavedra-Lozano J, Mejías A, Ahmad N, Peromingo E, Ardura Ml, Guillen $S$, et al. : Changing trends in acute osteomyelitis in children: impact of methicillin-resistant staphylococcus aureus infections. J Pediatr Orthop 28 : 569-575, 2008

24. Smith AS, Weinstein MA, Mizushima A, Coughlin B, Hayden SP, Lakin $M M$, et al. : MR imaging characteristics of tuberculous spondylitis vs vertebral osteomyelitis. AJR Am J Roentgenol 153 : 399-405, 1989

25. Tins BJ, Cassar-Pullicino VN : MR imaging of spinal infection. Semin Musculoskelet Radiol 8 : 215-229, 2004

26. Yoon YK, Jo YM, Kwon HH, Yoon HJ, Lee EJ, Park SY, et al. : Differential diagnosis between tuberculous spondylodiscitis and pyogenic spontaneous spondylodiscitis: a multicenter descriptive and comparative study. Spine J 15 : 1764-1771, 2015

27. Zilkens KW, Peters KM, Schwanitz BM : New inflammation markers for early detection of spondylodiscitis. Eur Spine J 1 : 152-155, 1992 